This item was submitted to Loughborough's Research Repository by the author.

Items in Figshare are protected by copyright, with all rights reserved, unless otherwise indicated.

\title{
OFDM joint data detection and phase noise cancellation for constant
} modulus modulations

PLEASE CITE THE PUBLISHED VERSION

http://dx.doi.org/10.1109/TSP.2009.2018362

PUBLISHER

(C) IEEE

VERSION

AM (Accepted Manuscript)

LICENCE

CC BY-NC-ND 4.0

REPOSITORY RECORD

Gong, Yu, and Xia Hong. 2019. "OFDM Joint Data Detection and Phase Noise Cancellation for Constant Modulus Modulations”. figshare. https://hdl.handle.net/2134/25671. 


\title{
OFDM Joint Data Detection and Phase Noise Cancellation for Constant Modulus Modulations
}

\author{
Yu Gong and Xia Hong \\ School of Systems Engineering \\ The University of Reading, Reading \\ RG6 6AY, UK \\ E-mail: $\{y \cdot g o n g, x \cdot h o n g\}$ ereading $\cdot$ ac $\cdot$ uk \\ Tel: +44 (0) 118378 8581, Fax: +44 (0) 1183788583
}

\begin{abstract}
This paper proposes a new algorithm for the OFDM joint data detection and phase noise (PHN) cancellation for constant modulus modulations. We highlight that it is important to address the overfitting problem since this is a major detrimental factor impairing the joint detection process. In order to attack the overfitting problem we propose an iterative approach based on minimum mean square prediction error (MMSPE) subject to the constraint that the estimated data symbols have constant power. The proposed constrained MMSPE algorithm (C-MMSPE) significantly improves the performance of existing approaches with little extra complexity being imposed. Simulation results are also given to verify the proposed algorithm.
\end{abstract}

\section{INTRODUCTION}

The phase noise (PHN) in an orthogonal frequency division multiplexing (OFDM) system arises from the imperfections at the receiver's oscillator, damaging the orthogonality among subcarriers [1], [2]. A typical PHN consists of two parts: the common PHN and the random PHN [3]. Most existing PHN cancellation algorithms (e.g. [3], [4]) mainly consider the common PHN which is an averaging effect over the OFDM transmission and can be mitigated with the help of pilot symbols. The random PHN, on the other hand, is much more difficult to handle as it varies from one symbol to another even for slowly fading channels. Recently, a family of algorithms for joint data detection and PHN cancellation based on the probabilistic approach of variational inference have been proposed [5].

In general, a joint estimation process may suffer from the "overfitting" problem so that the estimate is too close to the received samples and fits into the noise. The overfitting problem is particularly serious in the joint OFDM data detection and PHN cancellation. This is because for an OFDM symbol with $N$ subcarriers, there 
are $N$ data symbols and $N$ PHN to be determined from $2 N$ observations including $N$ from the receiving data and another $N$ from the PHN model. On another front, the PHN must be mitigated at every symbol since it varies from one symbol to another. This describes a very special case of parameter estimation problem: unlike the classic parameter estimation whereby the estimates can be improved by increasing the number of data samples, here the number of unknown parameters (i.e. the symbols and PHN) always equals the number of the "observation" samples, making it particularly vulnerable to overfitting which thus must be carefully handled as otherwise the whole joint process may be invalidated.

We note that, although it was not explicitly identified, the algorithms described in [5] are in fact equivalent to the Bayesian regularization utilizing the Gaussian distributions as the priors, a common method to combat overfitting [6]. This however may not be sufficiently effective for the OFDM PHN cancellation. In our recent paper [7] and further in [8], we proposed a new joint data detection and PHN cancellation algorithm based on minimum mean square prediction error (MMSPE), where the hard decision is applied to the symbol estimates at the end of each iteration. The hard decision process can effectively filter the noise out of the symbol estimates and remove the associated uncertainties due to the overfitting which would otherwise be carried forward over the iterations. However, the hard decision imposes as a non-linear constraint on the iterative procedure which may sometimes be too strong such that some symbol estimates are forced into the wrong direction over the iterations, resulting in performance loss. As will be shown in the simulation later in this paper, the MMSPE algorithm has close performance to, if not worse than, those proposed in [5] when the SNR is high (in which case there is little noise to be removed and the negative effect of the hard decision becomes more dominant). This motivates us to explore new methods to combat overfitting problem.

In this paper, we focus on the OFDM system with constant modulus modulations such as the PSK. Embedding the deterministic a priori information from the modulation that the data symbol must have constant power into the MMSPE cost function as a constraint, we propose a constrained MMSPE (C-MMSPE) algorithm to jointly detect the data symbol and cancel the PHN. The C-MMSPE algorithm can better handle the overfitting and has significantly superior performance to both the MMSPE algorithm and the algorithms described in [5]. The idea of using constant modulus has been well understood in many communications applications such as the Godard blind equalization [9], but this is the first time to be applied to the OFDM PHN cancellation. Although 
in general any prior information about the system, especially deterministic knowledge, should greatly help to improve the modelling performance, it usually leads to some complicated constrained optimization problems with large computational complexity. Luckily in the case of the OFDM PHN cancellation, we will show that the derived C-MMSPE has negligible additional complexities involved, in comparison to the unconstrained algorithms including the MMSPE algorithm in [7] and the algorithms described in [5]. The main contribution of this paper, over our previous work in [7], is the use of the constant modulus constraint to combat overfitting.

Similar to many existing approaches (e.g in [5]), this paper assumes the channel coefficients and noise variance are a priori known not only for simplicity but also to isolate the effect of the PHN cancellation. In practice, the channel estimation is necessary and actually becomes more difficult in presence of the phase noise. More discussion of joint channel estimation and phase noise cancellation can be found in literatures (e.g [10]). Another issue raised by one of the reviewers of this paper is the channel coding across multiple sub-carriers which can to some extent help cope with the PHN. Discussion about the joint decoding and PHN cancellation can be found in literatures (e.g. [11]). Finally, we assume in this paper that the PHN is sufficiently small as otherwise it is beyond the capability of any existing signal processing approaches to cancel. Due to the effectiveness of the hardware implementation, fortunately, the PHN is usually small in practice.

In this paper, we use ${ }^{*}$ to denote the conjugate of a complex signal, ${ }^{\mathrm{H}}$ to denote conjugate transpose, and $|x|=\sqrt{x^{*} \cdot x}$ to represent the norm of a signal $x$.

\section{SYSTEM MODEL}

For an OFDM system with $N$ subcarriers, the transmitted baseband OFDM signal can be written as:

$$
s(t)=\frac{1}{\sqrt{N}} \sum_{k=0}^{N-1} S_{k} e^{j 2 \pi k t / T}, \quad 0 \leq t \leq T
$$

where $T$ is the OFDM symbol period, and $S_{k}$ is the $k$ th data symbol which is modulated by a constant modulus modulation such that $\left|S_{k}\right|^{2}=E_{s}$ for all $k$, and $E_{s}$ is the symbol power. For better exposition, we assume the OFDM cyclic prefix is longer than the channel spread so that it can be clearly removed at the receiver without causing any intersymbol interference. We also assume the channel is slow fading that the channel coefficients remain unchanged within one OFDM symbol period. 
With the presence of the PHN, the received signal sequence becomes:

$$
r_{m}=\frac{1}{\sqrt{N}} e^{j \theta_{m}} \sum_{k=0}^{N-1} h_{k} S_{k} e^{j 2 \pi k m / N}+\eta_{m}, \quad m=0, \cdots, N-1
$$

where $h_{k}$ is the channel frequency response at subcarrier $k, \eta_{m}$ is the complex white noise with mean zero and variance $\sigma^{2}$, and $\theta_{m}$ is the $m$ th sample of the PHN. Since the channel coefficients are assumed to be known in this paper, the task is to jointly detect $S_{m}$ and $\theta_{m}$ given $r_{m}$ and $h_{m}$ for $m=0, \cdots, N-1$.

There are two types of PHN, namely the Wiener PHN and the Gaussian PHN ( [2], [5], [12]). Both can be represented in a general form of:

$$
\theta_{m}=\sum_{i=1}^{K} a_{i} \theta_{m-i}+\varepsilon_{m}, \quad m=0, \ldots, N-1
$$

where $\varepsilon_{m}$ is a zero mean white noise sequence with variance $\sigma_{\varepsilon}^{2}, K$ is the order size and $a_{i}$ is the $i$ th regressive factor. For the Wiener PHN, we have $K=1$ and $a_{1}=1$ in (3) so that it forms a random walk process which is nonstationary. While for the Gaussian PHN, $K$ is generally larger than one, and the polynomial $D(z)=1-\sum_{i=1}^{K} a_{i} z^{-i}$ has all roots inside the unit circle, corresponding to a stationary autoregressive (AR) process. Particularly for the Gaussian PHN, the coefficients $a_{i}$ and the order $K$ can either be estimated by applying the least squares (LS) and the Akaike's Information Criterion (AIC) on the PHN samples $\theta_{m}$ [13], or be obtained from the correlation matrix of the Gaussian PHN matrix which is assumed to be known [5].

\section{Joint Data Detection \& PHN CANCELlation}

\section{A. Constrained Minimum Mean Square Prediction Error}

From (2) and (3), a set of consistent model parameter estimates for $\theta_{m}$ and $S_{k}$ can be obtained from:

$$
\left\{\begin{array}{l}
r_{m}=\frac{1}{\sqrt{N}} e^{j \hat{\theta}_{m}} \sum_{k=0}^{N-1} h_{k} \hat{S}_{k} e^{j 2 \pi k m / N}+\xi_{m}, \quad m=0, \ldots, N-1 \\
\hat{\theta}_{m}=\sum_{i=1}^{K} a_{i} \hat{\theta}_{m-i}+\omega_{m}, \quad m=0, \ldots, N-1
\end{array}\right.
$$

where $\hat{\theta}_{m}, \hat{S}_{k}$ are the estimates of $\theta_{m}$ and $S_{k}$ respectively, $\xi_{m}$ and $\omega_{m}$ refer to the prediction errors of the channel model and the phase model respectively.

Letting $\hat{\mathbf{s}}=\left[\hat{S}_{0}, \cdots, \hat{S}_{N-1}\right]^{\mathrm{T}}, \boldsymbol{r}=\left[r_{0}, \cdots, r_{N-1}\right]^{\mathrm{T}}$ and the channel model prediction error vector be 
$\boldsymbol{\xi}=\left[\xi_{0}, \cdots, \xi_{N-1}\right]^{\mathrm{T}}$, we obtain a vector representation of the first equation of (4) as:

$$
\mathbf{r}=\operatorname{diag}\left\{e^{j \hat{\theta}_{0}}, \cdots, e^{j \hat{\theta}_{N-1}}\right\} \cdot \mathbf{P} \cdot \operatorname{diag}\left\{h_{0}, \cdots, h_{N-1}\right\} \cdot \hat{\mathbf{s}}+\boldsymbol{\xi}
$$

where $\mathbf{P}$ is the IFFT matrix with $\mathbf{P}^{\mathrm{H}} \mathbf{P}=\mathbf{I}$ and $p_{m, k}=\frac{1}{\sqrt{N}} e^{j(2 \pi(m-1)(k-1)) / N}$ which is the $m$ th row and $k$ th column element of $\mathbf{P}$, and $\mathbf{I}$ is an identical matrix with appropriate dimension.

Similar to that [7], we use the Pade approximation:

$$
e^{j \hat{\theta}_{m}} \approx \frac{2+j \hat{\theta}_{m}}{2-j \hat{\theta}_{m}}
$$

to replace the term $e^{j \hat{\theta}_{m}}$, so that we have:

$$
\xi_{m}=\frac{\left(2-j \hat{\theta}_{m}\right) r_{m}}{2}-\frac{1}{2 \sqrt{N}}\left(2+j \hat{\theta}_{m}\right) \sum_{k=0}^{N-1} h_{k} \hat{S}_{k} e^{j 2 \pi k m / N}+j \hat{\theta}_{m} \xi_{m} / 2
$$

Further denoting $\boldsymbol{\theta}=\left[\hat{\theta}_{0}, \cdots, \hat{\theta}_{N-1}\right]^{\mathrm{T}}$ and letting the phase model prediction error vector be $\boldsymbol{\omega}=$ $\left[\omega_{0}, \cdots, \omega_{N-1}\right]^{\mathrm{T}}$, we represent (7) and the second equation of (4) in vector forms as:

$$
\left\{\begin{array}{l}
\boldsymbol{\xi}=\mathbf{z}-\mathbf{Q} \boldsymbol{\theta}-\mathbf{Q}_{\xi} \boldsymbol{\theta} \\
\boldsymbol{\omega}=\boldsymbol{\Phi} \boldsymbol{\theta}
\end{array}\right.
$$

respectively, where

$$
\mathbf{z}=\left[\begin{array}{c}
r_{0}-\frac{1}{\sqrt{N}} \sum_{k=0}^{N-1} h_{k} \hat{S}_{k} \\
\vdots \\
r_{N-1}-\frac{1}{\sqrt{N}} \sum_{k=0}^{N-1} h_{k} \hat{S}_{k} e^{j 2 \pi k(N-1) / N}
\end{array}\right]
$$

$\mathbf{Q}_{\xi}=\operatorname{diag}\left\{\left(-j \xi_{1}\right) / 2, \cdots,\left(-j \xi_{N-1}\right) / 2\right\}$ which includes the terms of the channel model prediction error, $\mathbf{Q}=\operatorname{diag}\left\{q_{0}, \cdots, q_{N-1}\right\}$ which is the prediction errors free matrix with

$$
q_{m}=\frac{j}{2}\left[\sum_{k=0}^{N-1} h_{k} \hat{S}_{k} e^{j 2 \pi k m / N} / \sqrt{N}+r_{m}\right], \quad m=0, \cdots, N-1
$$


where:

$$
\boldsymbol{\Phi}=\left[\begin{array}{ccccc}
1 & 0 & \ldots & 0 & 0 \\
-a_{1} & 1 & 0 & 0 & 0 \\
-a_{2} & -a_{1} & 1 & 0 & 0 \\
\ldots & \ldots & \ldots & \ldots & \ldots \\
0 & 0 & -a_{K} & \ldots-a_{1} & 1
\end{array}\right]
$$

In order to jointly estimate the data symbol and the PHN, we construct a cost function based on MMSPE from both the channel and PHN models:

$$
\begin{aligned}
J & =E\left[\xi_{m}^{\mathrm{H}} \xi_{m}+\left(\sigma^{2} / \sigma_{\varepsilon}^{2}\right) \omega_{m}^{\mathrm{H}} \omega_{m}\right] \\
& \approx \frac{1}{N}\left[\boldsymbol{\xi}^{\mathrm{H}} \boldsymbol{\xi}+\left(\sigma^{2} / \sigma_{\varepsilon}^{2}\right) \boldsymbol{\omega}^{\mathrm{H}} \boldsymbol{\omega}\right] .
\end{aligned}
$$

Without losing any generality, furthermore, we assume the channel coefficients are normalized to unit such that $\left|h_{1}\right|^{2}+\cdots\left|h_{N}\right|^{2}=1$. For the constant modulus modulation, we have $\left|S_{k}\right|^{2}=E_{s}$. This extra a priori information from the constant modulus of the symbol estimates is then embedded into the joint estimation to form a constrained optimization problem:

$$
\left\{\begin{array}{l}
\min _{\hat{\mathbf{s}}, \boldsymbol{\theta}}\left\{\boldsymbol{\xi}^{\mathrm{H}} \boldsymbol{\xi}+\left(\sigma^{2} / \sigma_{\varepsilon}^{2}\right) \boldsymbol{\omega}^{\mathrm{H}} \boldsymbol{\omega}\right\} \\
\text { s.t. }\left|\hat{S}_{k}\right|^{2}=E_{s}, \quad k=0, \cdots, N-1 .
\end{array}\right.
$$

In theory, there exist local minima for the cost function of (13) which is not convex due to the non-linear nature of the phase noise term. When the phase noise is sufficiently small, however, (13) is locally convex and an iterative approach can be applied to search for the optimum solution. Fortunately in practice, the phase noise is usually small thanks to the effectiveness of the local oscillators, as otherwise the phase noise error is too large to be canceled by signal processing approaches.

\section{B. Iterative Approach}

The Lagrangian expression for the constrained optimization problem in (13) is formulated as:

$$
\mathcal{L}=\boldsymbol{\xi}^{\mathrm{H}} \boldsymbol{\xi}+\left(\sigma^{2} / \sigma_{\varepsilon}^{2}\right) \boldsymbol{\omega}^{\mathrm{H}} \boldsymbol{\omega}+\sum_{k=0}^{N-1} \lambda_{k}\left[\left|\hat{S}_{k}\right|^{2}-E_{s}\right]
$$


where $\lambda_{k}$ is the $k$ th Lagrange multiplier needed to be updated iteratively. Since $\mathcal{L}$ is quadratic with respect to $\mathbf{s}$ for a fixed $\boldsymbol{\theta}$, or vice versa, it can be reduced by setting $\frac{\partial}{\partial \hat{\mathbf{s}}} \mathcal{L}=\mathbf{0}$ and $\frac{\partial}{\partial \boldsymbol{\theta}} \mathcal{L}=\mathbf{0}$ alternatively.

First, setting $\frac{\partial}{\partial \boldsymbol{\theta}} \mathcal{L}=\mathbf{0}$ and making use of (8) yields:

$$
\left(\mathbf{Q}^{\mathrm{H}}+\mathbf{Q}_{\xi}^{\mathrm{H}}\right)\left(\mathbf{z}-\mathbf{Q} \boldsymbol{\theta}-\mathbf{Q}_{\xi} \boldsymbol{\theta}\right)-\frac{\sigma^{2}}{\sigma_{\varepsilon}^{2}} \boldsymbol{\Phi}^{\mathrm{H}} \boldsymbol{\Phi} \boldsymbol{\theta}=\mathbf{0} .
$$

Taking the expected value in terms of channel noise $\xi_{m}$ on (15) gives:

$$
\left(\mathbf{Q}^{\mathrm{H}} \mathbf{Q}+\frac{\sigma^{2}}{\sigma_{\varepsilon}^{2}} \boldsymbol{\Phi}^{\mathrm{H}} \boldsymbol{\Phi}\right) \boldsymbol{\theta}=\mathbf{Q}^{\mathrm{H}} \mathbf{z}-\frac{j \sigma^{2}}{2} \mathbf{1}
$$

where $\mathbf{1}$ is a vector with all elements as 1 , and it is assumed that at optimality $E\left(\mathbf{Q}_{\xi}^{\mathrm{H}} \mathbf{Q}\right)=\mathbf{0}, E\left(\mathbf{Q}_{\xi}^{\mathrm{H}} \mathbf{Q}_{\xi}\right)=$ $\sigma^{2} \mathbf{I} / 4$ and $E\left(\mathbf{Q}_{\xi}^{\mathrm{H}} \mathbf{z}\right)=E\left(\mathbf{Q}_{\xi}^{\mathrm{H}} \mathbf{Q}_{\xi}\right) \boldsymbol{\theta}+E\left(\mathbf{Q}_{\xi}^{\mathrm{H}} \boldsymbol{\xi}\right)=\sigma^{2} \boldsymbol{\theta} / 4-j \sigma^{2} \mathbf{1} / 2$

Similarly letting $\frac{\partial}{\partial \hat{\mathbf{s}}} \mathcal{L}=\mathbf{0}$ and making use of (5) gives:

$$
\hat{\mathbf{s}}=\operatorname{diag}\left\{\frac{h_{0}^{*}}{\left|h_{0}\right|^{2}+\lambda_{0}}, \cdots, \frac{h_{N-1}^{*}}{\left|h_{N-1}\right|^{2}+\lambda_{N-1}}\right\} \cdot \mathbf{b}
$$

where

$$
\mathbf{b}=\mathbf{P}^{\mathrm{H}} \operatorname{diag}\left\{e^{-j \hat{\theta}_{0}}, \cdots, e^{-j \hat{\theta}_{N-1}}\right\} \mathbf{r} .
$$

Rewriting (17) in a scalar form gives:

$$
\hat{S}_{k}=\frac{h_{k}^{*} b_{k}}{\left|h_{k}\right|^{2}+\lambda_{k}}, \quad k=0, \cdots, N-1
$$

where $b_{k}$ is the $k$ th element of $\mathbf{b}$. Finally substituting (19) into the constraint $\left|\hat{S}_{k}\right|^{2}=E_{s}$ gives the update of the Lagrange multipliers ${ }^{1}$ :

$$
\lambda_{k}=\frac{1}{\sqrt{E_{s}}}\left|h_{k}^{*} b_{k}\right|-\left|h_{k}\right|^{2}, \quad k=0, \cdots, N-1
$$

The solutions of (16), (17) and (20) form the iterative procedure for the joint data detection and PHN cancellation. It can be seen from (19) that the Lagrange multiplier, $\lambda_{k}$, can be regarded as a special parameter regularization factor to overcome the overfitting. As was stated in the Introduction, the algorithms in [5]

${ }^{1}$ There exists another solution: $\lambda_{k}^{\prime}=-\frac{1}{\sqrt{E_{s}}}\left|h_{k}^{*} b_{k}\right|-\left|h_{k}\right|^{2}$. It is obvious $\left|\lambda_{k}^{\prime}\right|>\left|\lambda_{k}\right|$. Since the Lagrange multiplier can be regarded 
effectively applied the parameter regularization factor to overcome the overfitting. There are however basic differences between the two approaches: First, the regularization factor for the algorithms in [5] was based on a statistical prior information that the channel noise and PHN are Gaussian, while $\lambda_{k}$ in the above proposed approach is derived from the constant modulus constraint which is a deterministic prior information. It is intuitive that deterministic prior information leads to better performance than the statistical one. Secondly, rather than having a single and fixed regularization factor for every symbol estimate in [5], the proposed algorithm applies multiple and adjustable regularization factors for different symbol estimate, or equivalently, $\lambda_{i} \neq \lambda_{j}$ for $i \neq j$. This makes the proposed algorithm much more effective in handling the overfitting.

As was suggested by one of the reviewers of this paper, the above approach can be further simplified by substituting (20) into (19) so that:

$$
\hat{S}_{k}=\sqrt{E_{s}} \cdot \frac{\tilde{S}_{k}}{\left|\tilde{S}_{k}\right|}, \quad k=0, \cdots, N-1
$$

where $\tilde{S}_{k}=h_{k}^{-1} b_{k}$ which is actually an intermediate estimate of the data $S_{k}$ with the estimated PHN being removed. This gives an interesting insight into the proposed approach: the effect of the constant modulus constraint is equivalent to normalizing the magnitude of each estimated data to the constant modulus before they move forward to the next iteration.

\section{The Algorithm}

The algorithm of the C-MMSPE algorithm for the joint data detection and PHN cancellation is summarized as below, where the superscript ${ }^{(l)}$ denotes the corresponding parameters at the $l$ th iteration, $\boldsymbol{\Phi}$ is given by (11), $\mathbf{P}$ is the IFFT matrix, and accordingly $\mathbf{Q}$ and $\mathbf{z}$ defined in (10) and (9) are denoted as $\mathbf{Q}\left(\hat{\mathbf{s}}^{(l-1)}\right)$ and $\mathbf{z}\left(\hat{\mathbf{s}}^{(l-1)}\right)$ respectively.

Initialization

$$
\hat{\mathbf{s}}^{(0)}=\operatorname{diag}\left\{h_{1}^{-1}, \cdots, h_{N-1}^{-1}\right\} \mathbf{P}^{\mathrm{H}} \mathbf{r}
$$

Replace $\hat{\mathbf{s}}^{(0)}$ by its hard decision 


$$
\begin{aligned}
& \text { For } \begin{aligned}
l= & 1,2,3, \cdots \\
\boldsymbol{\theta}^{(l)} & =\left(\left[\mathbf{Q}\left(\hat{\mathbf{s}}^{(l-1)}\right)\right]^{\mathrm{H}} \mathbf{Q}\left(\hat{\mathbf{s}}^{(l-1)}\right)+\frac{\sigma^{2}}{\sigma_{\varepsilon}^{2}} \boldsymbol{\Phi}^{\mathrm{H}} \mathbf{\Phi}\right)^{-1} \cdot \operatorname{Re}\left\{\left[\mathbf{Q}\left(\hat{\mathbf{s}}^{(l-1)}\right)\right]^{\mathrm{H}} \mathbf{z}\left(\hat{\mathbf{s}}^{(l-1)}\right)\right\} \\
\mathbf{b}^{(l)} & =\mathbf{P}^{\mathrm{H}} \operatorname{diag}\left\{e^{-j \hat{\theta}_{0}^{(l)}}, \cdots, e^{-j \hat{\theta}_{N-1}^{(l)}}\right\} \mathbf{r} \\
\hat{S}_{k} & =\sqrt{E_{s}} \cdot \frac{h_{k}^{-1} b_{k}}{\left|h_{k}^{-1} b_{k}\right|}, \quad k=0, \cdots, N-1
\end{aligned}
\end{aligned}
$$

End

In the C-MMSPE algorithm, the symbol estimate $\hat{\mathbf{s}}^{(0)}$ is initialized as the output of the traditional OFDM detector with the PNH being ignored. This makes the iterative procedure begin at a fair good level so that the convergence can be reached. For a better initialization, a hard decision procedure can also be applied to $\hat{\mathbf{s}}^{(0)}$ to remove the noise from the initial stage. We particularly point out that, however, the hard decision has not been applied within the iterations in C-MMSPE as otherwise the C-MMSPE and the MMSPE algorithms ( [7]) have close performance. This is because once the hard decision is applied, the constraint of the constant modulus is automatically satisfied, but not vice versa. Thus the hard decision is a stronger constraint than the constant modulus and will override the latter.

Comparing the C-MMSPE and MMSPE algorithms shows that they have almost identity computational complexity, except that the C-MMSPE algorithm needs to normalize the data estimate which only requires a group of simple scalar calculations (Eq. (24)). Similar to that in [7], fast algorithm based on Givens rotation can be applied to calculate the matrix inversion in (22), the main complexity of the iterative approach. It has been shown in [7] that the Givens Rotation based fast algorithm not only is more robust but also has less complexity than the conjugate gradient (CG) method used in [5].

\section{NUMERICAL Simulation}

In this section, we compare the proposed C-MMSPE algorithm with the MMSPE algorithm ( [7]) and the ICM algorithm described in [5]. Since the comparison based on the Wiener PHN and Gaussian PHN shows similar results, only the results for the Wiener PHN are reported here due to the space constraint of this paper. The parameters of the simulation system are set as follows:

1) An OFDM symbol size of 64 subcarriers with each subcarrier modulated by 16-PSK;

2) A Rayleigh mutlipath fading channel with a delay of 10 taps and an exponentially decreasing power delav profile having a decav constant of 3 tap; 
3) The Wiener PHN generated by the random walk described in (3) with the standard deviation of $\varepsilon_{m}$ being at $\sigma_{\varepsilon}=0.75^{\circ}$.

4) Four iterations applied for all of the iterative algorithms including the C-MMSPE, MMSPE and the ICM algorithms.

Fig. 1 compares the BER performance for different approaches, where the hard decision is applied on the symbol estimate at the initial stage for all of the three algorithms. For better comparison, the BER curves for the classic OFDM detector with the PHN being ignored and the detector with perfect PHN cancellation are also plotted. It is shown that the MMSPE algorithm has better performance than the ICM algorithm when the SNR is smaller than $32 \mathrm{~dB}$, but performs slightly worse than the latter when the SNR is higher than $32 \mathrm{~dB}$. This observation well matches our previous statement that the hard-decision is sometimes too strong a constraint that "drags" the iteration into the wrong directions especially when the SNR is very high. Among all of the approaches, the C-MMSPE algorithm has obviously the best performance such that it has similar performance to the MMSPE algorithm when the SNR is low (e.g SNR $<24 \mathrm{~dB}$ ), but has significantly better performance than the other two when the SNR is high (e.g SNR $>24 \mathrm{~dB})$.

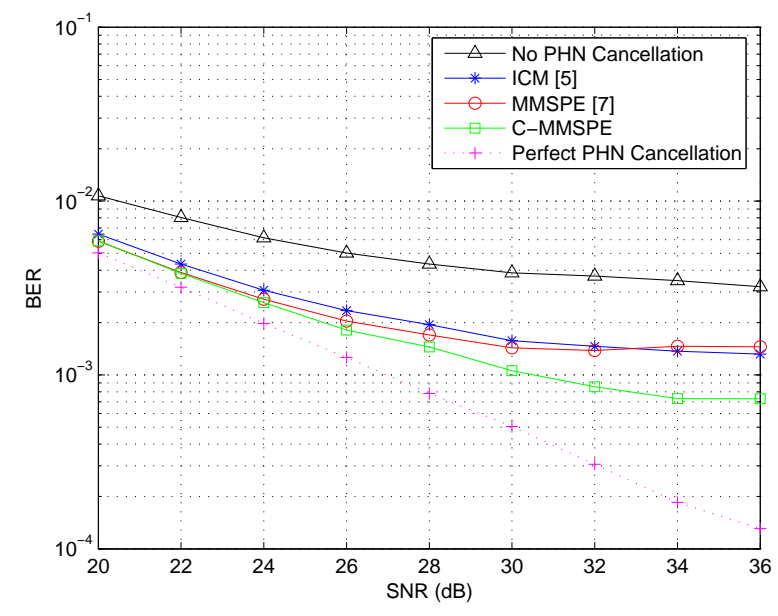

Fig. 1. BER performance comparison, where channel information is assumed to be a priori known.

We highlighted before that, in the proposed C-MMSPE approach, the hard decision is only applied at the initialization stage but not within the iterations, because the hard decision as a stronger constraint will override the constant modulus constraint. The numerical simulation does verify this statement that the C-MMSPE and MMSPE algorithms have identical BER performance if the hard decision is applied at every iteration. The 
results are not shown here in this short paper. This further confirms that the constant modulus constraint can better handle the overfitting than the hard decision procedure.

In the next experiment, we show how the above PHN cancellation approaches perform under the scenario that the channel coefficients are also estimated on-line. We consider a simple case here that the channel is estimated every 5 OFDM symbols (each OFDM symbol contains 64 data), and the channel coefficients remain unchanged between any two adjacent channel estimation events. Further for simplicity, the channel estimation is performed by regarding the PHN as noise. Fig. 2 shows the BER performance for different approaches. It is clear that the proposed C-MMSPE algorithm still has better performance than both the ICM and MMSPE algorithms, though the improvement becomes less significant due to the channel estimation error which can be regarded as another source of noise. This simple example further verifies the effectiveness of the proposed CMMSPE algorithm which does not collapse under the channel estimation error. In practice, rather than treating the PHN as noise, a joint approach of channel estimation and PHN cancellation is normally required so that the channel estimation would become much more accurate (e.g [10]). And we would then expect significantly better performance than that shown in Fig. 2. This topic is beyond the scope of this paper.

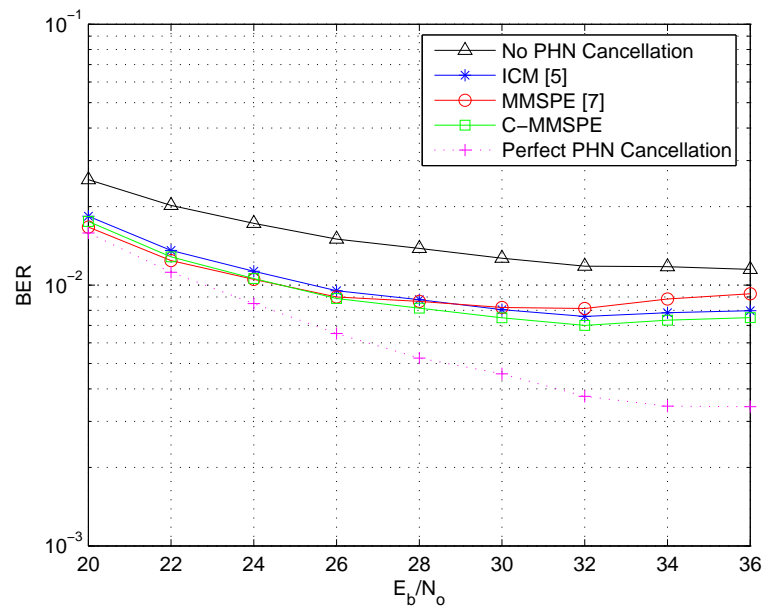

Fig. 2. BER performance comparison, where channel coefficients are estimated on-line.

\section{CONCLusions}

This paper proposed a new algorithm for joint OFDM symbol detection and PHN cancellation based on MMSPE cost function subject to the constant modulus constraint of the symbol estimate. The proposed C-MMSPE can better handle the overfitting problem than existing approaches, significantly improving the 
performance. Although the C-MMSPE algorithm was derived for constant modulus modulations, it is possible to be extended to more general modulation methods, and this is left as a future research topic.

\section{ACKNOWLEDGEMENT}

The authors would like to thank Dr D. D. Lin and Professor T. J. Lim from the University of Toronto for their kind help in setting up the the simulation parameters in this article. The authors are also thankful for the reviewers and editors for their constructive comments.

\section{REFERENCES}

[1] T. Pollet, M. V. Bladel, and M. Moeneclaey, "BER sensitivity of OFDM systems to carrier frequency offset and Wiener phase noise," IEEE Trans. on Commun., vol. 43, no. 2, pp. 191-193, Feb. 1995.

[2] T. Tomba, "On the effect of wiener phase noise in OFDM systems," IEEE Transactions on Communications, vol. 46, no. 5, pp. 580-583, 1998.

[3] K. Nikitopoulos and A. Polydoros, "Phase-impairment effects and compensation algorithms for OFDM systems," IEEE Trans. on Commun., vol. 53, no. 4, pp. 698-707, April 2005.

[4] S. Wu and Y. Bar-Ness, "OFDM systems in the presence of phase noise: consequences and solutions," IEEE Transactions on Communications, vol. 52, no. 11, pp. 1988-1996, 2004.

[5] D. D. Lin and T. J. Lim, “The variational inference approach to joint data detection and phase noise estimation," IEEE Transactions on Signal Processing, vol. 55, no. 5, pp. 1862-1874, 2007.

[6] D. J. C. MacKay, "Bayesian interpolation,” Neural Computation, vol. 4, no. 3, pp. 415-447, 1992.

[7] Y. Gong and X. Hong, "A new algorithm for OFDM joint data detection and phase noise cancellation," in IEEE International Conference on Communications (ICC 2008), Beijing, China, 19-23 May 2008.

[8] _ _ "Ofdm joint data detection and phase noise cancellation based on minimum mean square prediction error," Signal Processing, Elsevier, vol. 89, no. 4, pp. 502-509, April 2009.

[9] D. N. Godard, "Self-recovering equalization and carrier tracking in two-dimentional data communication systems," IEEE Signal Communications, vol. COM-28, no. 11, pp. 1867 - 1875, Nov. 1980.

[10] D. D. Lin, R. A. Pacheco, T. J. Lim, and D. Hatzinakos, "Joint estimation of channel response, frequency offset and phase noise in ofdm," IEEE Trans. on Signal Processing, vol. 54, no. 9, pp. 3542 - 3554, Sept. 2006.

[11] S. Bittner, W. Rave, and G. Fettweis, "Joint iterative transmitter and receiver phase noise correction using soft information," in IEEE International Conference on Communications, 2007 (ICC '07), 24-28 June 2007, pp. 2847 - 2852.

[12] L. Piazzo and P. Mandarini, "Analysis of phase noise effects in OFDM modems," IEEE Transactions on Communications, vol. 50, no. 10, pp. 1696-1705, 2002.

[13] H. Akaike, "A new look at the statistical model identification," IEEE Trans Automat. Contr, vol. AC-19, pp. 716 - 723, Dec. 1974. 